\title{
Upgrade of the Edge Charge Exchange Recombination Spectroscopy System at the High Field Side of ASDEX Upgrade
}

\author{
D.J. Cruz-Zabalaa,b, E. Viezzer,a,b, M. Grienerc, U. Plank ${ }^{c}$, M. Cavedon ${ }^{c}$, \\ P. Cano-Megias, ${ }^{\mathrm{e}, \mathrm{b}}, \mathbf{R}$. Dux ${ }^{\mathrm{c}}$, G. Fuchert ${ }^{\mathrm{d}}$, J. Garcia-Lopez ${ }^{\mathrm{a}, \mathrm{b}}, \mathrm{M}^{\prime}$ M. Garcia-Munoz, \\ A. Jansen van Vuuren ${ }^{c}, T$. Pütterich ${ }^{c}$, V. Rohde ${ }^{c}$ and ASDEX Upgrade team ${ }^{c}$ \\ ${ }^{a}$ Department of Atomic, Molecular and Nuclear Physics, University of Seville, \\ Avda. Reina Mercedes, 41012 Seville, Spain. \\ ${ }^{b}$ CNA (U. Sevilla, CSIC, J. de Andalucia), \\ Thomas Alva Edison, 7, 41092, Seville, Spain. \\ ${ }^{c}$ Max-Planck-Institut für Plasmaphysik, \\ Boltzmannstr. 2, 85748 Garching, Germany. \\ ${ }^{d}$ Max-Planck-Institut für Plasmaphysik, \\ Wendelsteinstr. 1, 17491 Greifswald, Germany. \\ ${ }^{e}$ Department of Energy Engineering, University of Seville, \\ Camino de los Descubrimientos, $s / n$. 41092, Seville, Spain. \\ E-mail: dcruz3@us.es
}

\section{ABSTRACT:}

The upgrade of the high field side (HFS) edge charge exchange recombination spectroscopy (CXRS) system of ASDEX Upgrade is presented. This diagnostic provides temperature, rotation and radiance measurements of impurity species by taking advantage of the gas puff based CXRS technique (GP-CXRS). The system is formed by a fast piezoelectric valve, that injects thermal neutrals into the plasma, and two optical heads. The localized gas injection together with properly aligned lines of sights (LOS) lead to a high spatial resolution of 5-19 mm. Fast gas puff modulation allows a precise subtraction of the passive part of the signal. The existing poloidal optical head has been replaced with a new one to increase the radial resolution. The number of lines of sight (LOS) of the poloidal optical head has been increased from 8 to 16 covering around $7 \mathrm{~cm}$ of the plasma edge at the HFS. The same radial range is also viewed by a toroidal optical head. The neutral deposition, needed to calculate the impurity density profile, has been modelled using the FIDASIM code. A realistic gas puff geometry has been implemented in the code. The first measurements of impurity temperature, rotation and radiance utilizing the upgraded diagnostic are presented.

KEYWORDS: Nuclear instruments and methods for hot plasma diagnostics; Plasma diagnostics charged-particle spectroscopy. 


\section{Contents}

\section{Introduction}

1.

2. The high field side charge exchange recombination spectroscopy system 1.

2.1 Fast in-vessel valve

2.1.1 Design

2.

2.1.2 Flowrate calibration

2.

2.2 Diagnostic optics

\section{First measurements}

4.

4. Gas puff modelling using FIDASIM

5.

5. Conclusions

6.

\section{Introduction}

The most common technique to measure impurity properties in magnetically confined fusion devices is charge exchange recombination spectroscopy (CXRS) [1]. This technique exploits the light emitted due to charge exchange reactions between neutral particles, that are injected via a neutral beam or a gas puff, and ionized plasma impurities:

$$
A^{Z+}+D \rightarrow A^{(Z-1)+*}+D^{+} \rightarrow A^{(Z-1)+}+\boldsymbol{h} \boldsymbol{v}+D^{+}
$$

After the reaction, the excited impurity atoms decay and emit photons that are collected by the optics of the diagnostic. The spectrum of the emitted light yields information on the observed species. The temperature and rotation are related to the Doppler width and shift, respectively, while the impurity density is related to the intensity of the signal.

Usually, the edge CXRS measurements are taken at the low field side (LFS) for technical simplicity, but several studies have shown that there are poloidal impurity asymmetries between the LFS and the high field side (HFS) [2, 3, 4]. At the HFS of a tokamak, the gas puff based CXRS (GP-CXRS) is the most common alternative to the neutral beam injection (NBI) based CXRS. Instead of injecting high energy neutrals, the GP-CXRS injects thermal neutrals and provides a localization at the edge of the plasma [5]. Other advantages of the GP-CXRS are the low cost and maintenance needed.

\section{The high field side charge exchange recombination spectroscopy system}

Earlier studies have shown differences in plasma profiles when comparing HFS and LFS measurements [2, 3, 4]. In order to study these differences in further detail, the HFS CXRS system of ASDEX Upgrade has been upgraded. The main goals of the upgrade are to provide a detailed characterization of the neutrals with improved resolution and a better background characterization. The upgraded system is formed by a new fast valve, a new poloidal optical head and a toroidal optical head. A scheme of the diagnostic set up is shown in figure 1(a). In the following, the different parts of the diagnostic are presented. 


\subsection{Fast in-vessel valve}

The fast in-vessel piezoelectric valve based on the design presented in [6] locally injects thermal gas in the plasma in order to produce charge exchange reactions. The valve is located inside a box that is used as a gas reservoir (gas box). The gas box is placed in the HFS of ASDEX Upgrade, behind the first wall.

\subsubsection{Design}

The new valve is based on a piezoelectric actuator which amplifies the voltage controlled piezo movement by a spring. Figures 1 (b) and 1(c) show a picture of the piezo element and a CAD drawing of the gas box. Due to the actuator geometry, the horizontal movement of the piezo is transformed into a vertical displacement. The piezo used is a PX500 [7] and it has a total stroke of $500 \mu \mathrm{m}$ and a voltage range of $-20 \mathrm{~V}$ to $130 \mathrm{~V}$. The valve is normally closed, in which a voltage increase leads to an elevation of the sealing plate so that gas can flow through the capillary into the plasma. Figure 2 shows pictures of the valve in open and closed position. During real experiments, the opening and closing of the valve is controlled by voltage pulses, the shortest pulse length allowed being $10 \mathrm{~ms}$. The opening and closing time of the valve is 2 ms approximately.
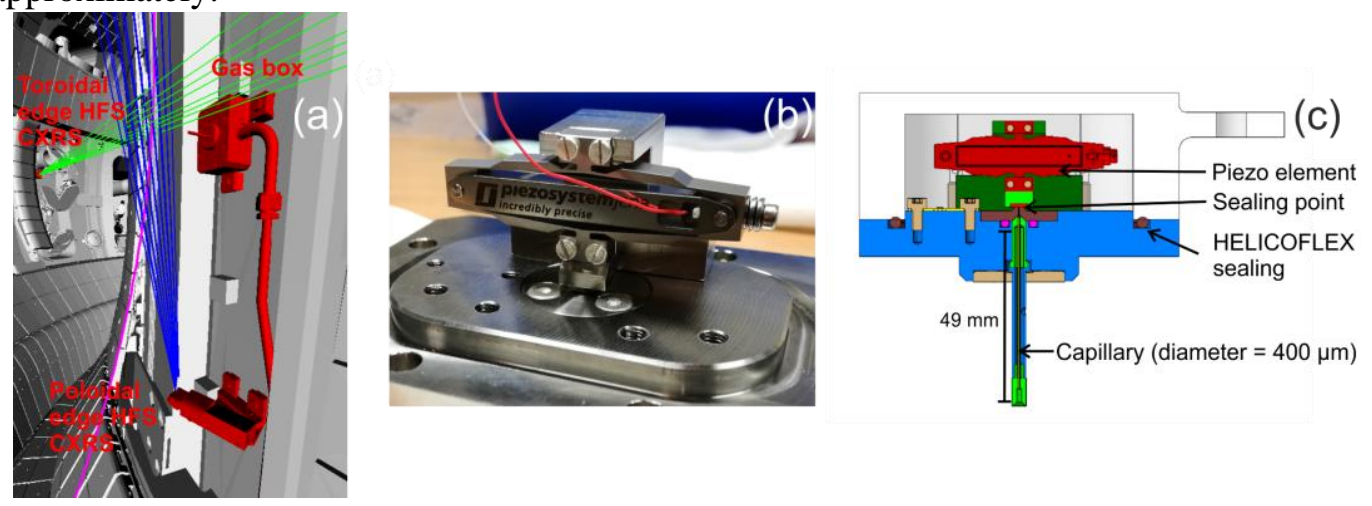

Figure 1. Overview of the HFS CXRS system (magenta line corresponds to the separatrix) (a), picture of the piezoelectric actuator (b) and sketch of the main parts of the gas box (c).

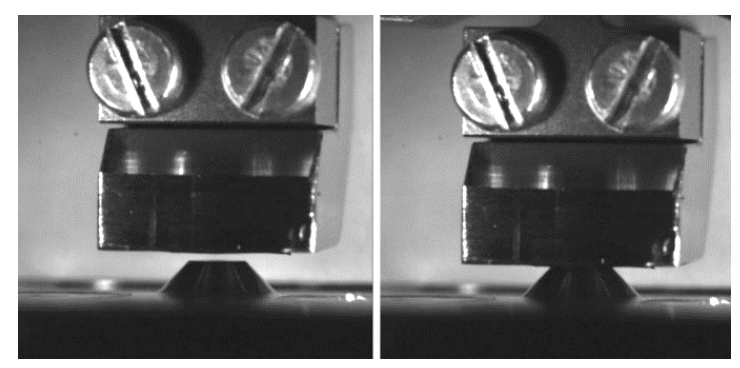

Figure 2. Valve opened (left) and closed (right).

\subsubsection{Flowrate calibration}

The neutrals flowrate can be set by adjusting the pressure inside the gas box. In order to know accurately the relation between the pressure in the gas box and the real flowrate, several calibrations have been performed for different gases in a test vacuum chamber. The calibration procedure is summarized in the following: 
1. A certain pressure $p_{\text {gas box }}$ is set inside the gas box.

2. The valve is opened for a certain time $\Delta t$.

3. The pressure increase $\Delta p_{\text {baratron }}$ is measured in the vacuum chamber by a baratron.

4. The ideal gas law is used for calculating the real flowrate:

$$
\dot{n}_{\text {gas box }}=\frac{\Delta p_{\text {baratron }} V}{\Delta t k_{\mathrm{B}} T}
$$

with $V$ and $T$ being the volume and the temperature of the vacuum chamber respectively, and $k_{\mathrm{B}}$ the Boltzmann constant. This procedure is repeated for different values of $p_{\text {gas box }}$ and different gases. The calibration curves are shown in figure 3. For deuterium, the calibration was obtained only up to 300 mbar because of limitations in the pumping system of the vacuum chamber. This limitation comes from the flowrate of deuterium that the pumps of the vacuum chamber can absorb. For higher pressures, a linear extrapolation is performed. Measurements of the deuterium flowrate in the vessel of ASDEX Upgrade show good agreement with the extrapolation in the calibration. In this case, a manometer was used to measure the increment of pressure due to the opening of the valve. Knowing the volume of the vessel, the flowrate can be estimated. For helium, an oscillating behaviour is observed at pressures higher than 4200 mbar while for nitrogen and argon, a plateau is observed at around $1500 \mathrm{mbar}$. These deviations from the linear dependence are still being investigated.

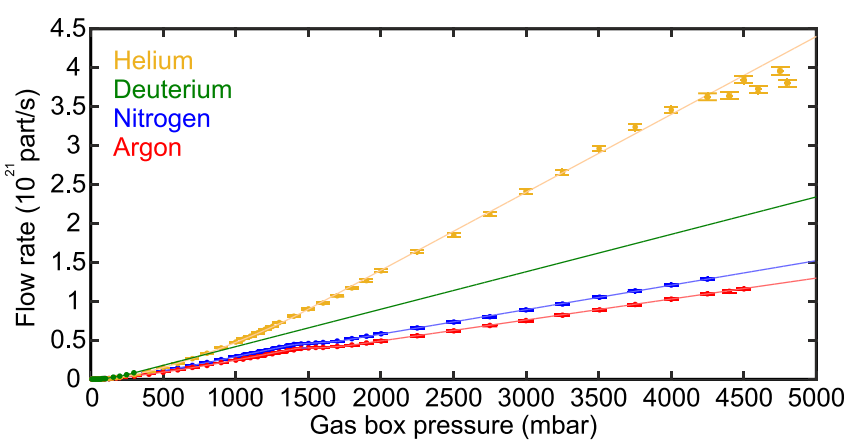

Figure 3. Calibration curves for different gases.

\subsection{Diagnostic optics}

The light emitted after the CX reactions between the injected neutrals and the impurities of the plasma is collected by the optical heads. The HFS CXRS diagnostic has two optical heads: one poloidally oriented and another one toroidally oriented. During this work, the poloidal optical head has been upgraded increasing the number of lines of sight (LOS) from 8 to 16, covering around $7 \mathrm{~cm}$ of the plasma edge. This upgrade has increased the resolution of the poloidal optical head to 5-19 mm depending on the line of sight. The toroidal optical head is covering the same range with a resolution of $10 \mathrm{~mm}$. Both optical heads are connected to a spectrometer by optical fibres with a diameter of $400 \mu \mathrm{m}$. The $f$ number of the lenses are 3.6 for the poloidal head and 3.7 for the toroidal head, while the focal length are $40 \mathrm{~mm}$ and $100 \mathrm{~mm}$ respectively. The optical heads were aligned to the injection point of the gas box using a 3D measurement device [8]. For this alignment, the optical fibres were backwards illuminated so that the geometry of the LOS was visible inside the torus. Figure 4 shows a top view of the poloidal optical head showing the possible movement for the alignment, $\alpha$ being the maximum angle that can be set. The mirror that transfers the light to the optical fibres can also be moved to 
improve the alignment. Two contributions have been considered for the calculation of the resolution: the LOS spot size at the injection point and the radial width of the gas cloud crossed by the LOS. After the alignment, an intensity calibration was performed using a standardized light source with a known spectral radiance. This calibration includes the in-vessel optics and the spectrometer. The spectrometer used for calibrating and operating the system has 24 channels, an exposure time of $2.2 \mathrm{~ms}$, a grating of 2400 lines $/ \mathrm{mm}$ and a wavelength resolution of $0.02 \mathrm{~nm}$. A wavelength calibration is performed after each discharge by means of a neon lamp.

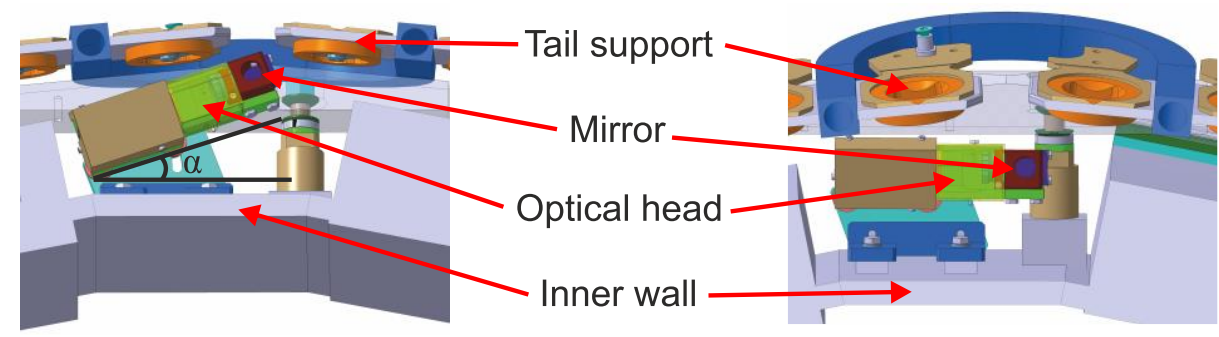

Figure 4. Drawings of the poloidal optical head showing the movement available for alignment. $\alpha$ is the maximun angle available for the alignment.

\section{First measurements}

The first measurements using the new diagnostic were obtained during an H-mode discharge with the following parameters: toroidal magnetic field of $2.5 \mathrm{~T}$, plasma current of $1 \mathrm{MA}$, ELM (Edge Localized Modes) frequency of $80 \mathrm{~Hz}$, NBI power of $5 \mathrm{MW}$ and ECRH (Electron Cyclotron Resonance Heating) power of 3.5 MW. The plasma was moved toward the HFS during one second in order to have more lines of sight inside the plasma, as the HFS CXRS system is measuring at the very edge of the plasma. The gas box was filled with deuterium at $1.2 \mathrm{bar}$, that corresponds to a flowrate of $5.16 \cdot 10^{20} \mathrm{part} / \mathrm{s}$. The valve was opened during 16 pulses of $20 \mathrm{~ms}$ when the plasma was close to the inner wall. The spectrometer was set to measure the $\mathrm{N}^{7+}$ line $(\mathrm{n}=9 \rightarrow 8)$ at $566.937 \mathrm{~nm}$. Figure 5 shows examples of active (valve open) and passive (valve closed) frames and the resulting frame after the background subtraction. The measured spectra are represented with black colour while the fit to the different lines are represented in red and blue. The total fit (magenta line) is the sum of the red gaussian, the blue gaussian and a baseline fit, which is an offset adjusted to the residual background. Note that the $\mathrm{N}^{2+}$ line is mostly passive, as it has almost the same intensity in the active and the passive frames. The overlap of the two lines shows the importance of characterizing the background properly. The temperature and rotation velocity can be derived from the Doppler width and shift of the signal, respectively. 

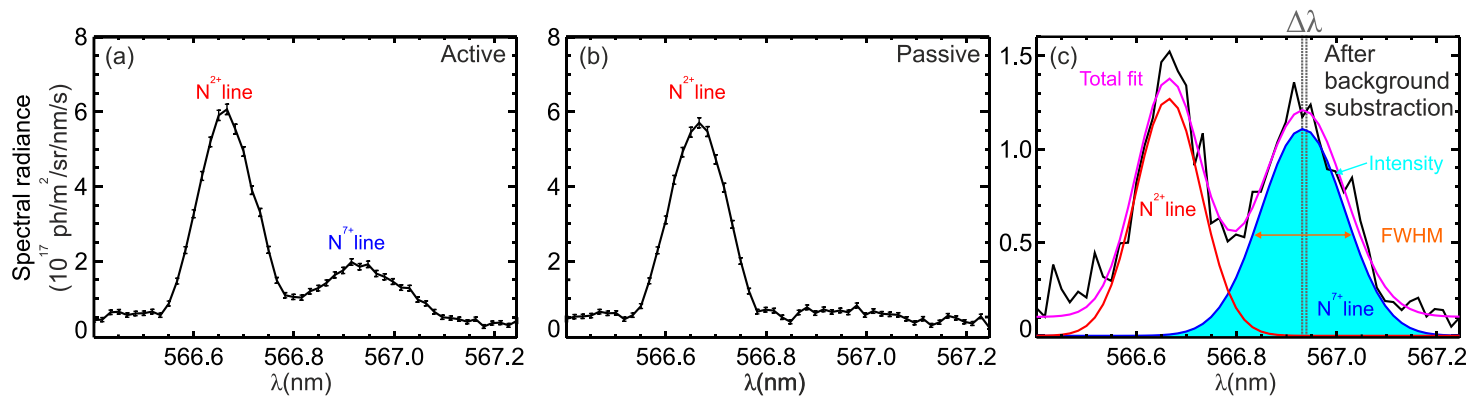

Figure 5. Active (a), passive (b) and background subtracted (c) spectra of the measured nitrogen lines. The background subtracted frame is the active frame minus the passive frame. Note the different scales. $\Delta \lambda$ corresponds to the Doppler shift of the signal.

The temperature, rotation and radiance profiles are shown in figure 6 . In the temperature plot, measurements of the LFS systems are also included for comparison. For these plots, the radial profile alignment technique is performed, being $3 \mathrm{~mm}$ the shift applied in both LFS and HFS systems. These shifts are applied to align impurity temperature with electron temperature and with separatrix position. The temperature profiles in the LFS and HFS match well, in agreement with previous observations [9]. The poloidal and toroidal impurity rotation in the HFS present different shapes, according to $[2,3]$. Note that time points affected by edge localized modes (ELMs) are removed from the analysis. Atomic physics effects might produce small deviations in the temperature and rotation measurements [10]. The calculation of the charge exchange cross-section effect for gas puff CXRS has been shown to be small with corrections of $\leq 15 \%$ [5]. In the case of the gyro-motion effect [11, 12], the corrections have been calculated for realistic values of the lifetime (0.5-2.0 ns) for the geometry of the HFS gas puff CXRS system. The corrections are $\leq 25 \%$ and are thus, not included in the standard analysis.
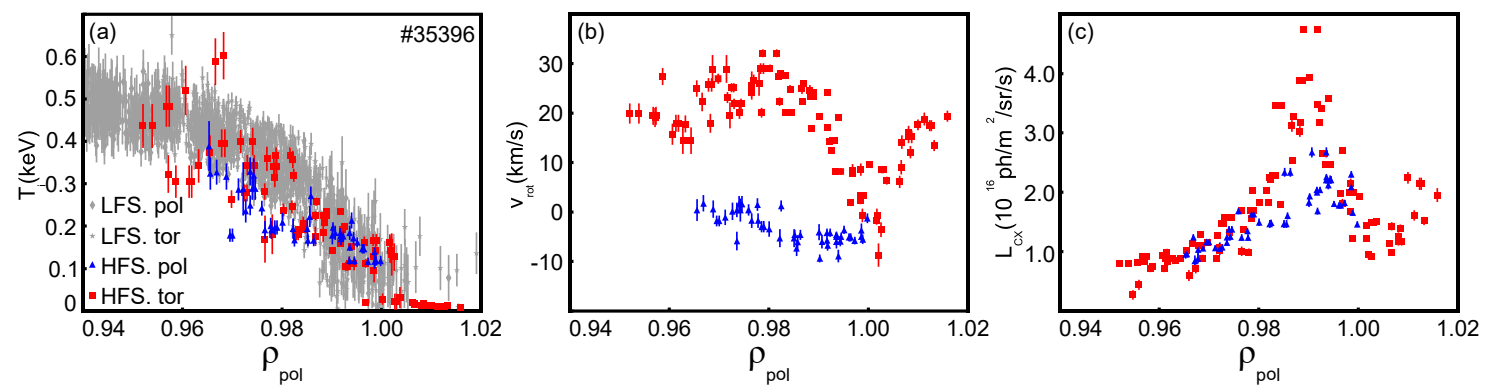

Figure 6. First experimental profiles obtained with the upgraded HFS CXRS system. Grey points on the temperature plot correspond to LFS CXRS systems. The dashed line corresponds to the separatrix.

\section{Gas puff modelling using FIDASIM}

Simulations of the gas puff neutral density distribution are key to determine the impurity density. During this work, FIDASIM [13,14] code has been used to model the HFS gas puff. FIDASIM is a 3D code that models density and spectra of the NBI, halo and FIDA (fast ion $\mathrm{D}_{\alpha}$ ) neutrals. FIDA neutrals are neutralized fast ions. For studying gas puff neutrals, a new model has been implemented in the code. This model launches neutrals from the HFS with thermal 
energy and a certain geometry distribution. The geometry distribution consists in a cone around the injection direction with an opening angle of $20^{\circ}$. This geometry was observed during a glow discharge in the vacuum chamber. In order to simplify the calculations, D atoms are considered instead of $\mathrm{D}_{2}$ molecules. This simplification has been done to test the model. A more realistic model should include molecular effects. After launching the neutrals, FIDASIM calculates the distribution of the first generation of neutrals and the distribution of halo neutrals. Halo neutrals are created when a gas puff neutral donates its electron to the thermal plasma (typically deuterium, instead of to an impurity). The halo produces a cloud of neutrals around the gas puff. FIDASIM gives also the distribution of neutrals in the different excited states. Note that neutrals in the first excited state $(n=2)$ give the main contribution in the CXRS signal [5]. Figure 7 shows a poloidal and a top view of the first generation of gas puff neutrals. These plots are obtained for the same shot as the experimental data shown in this paper. The black line shown in figure 7(a) and 7(b) corresponds to the separatrix. These simulations will make possible the calculation of the impurity density profile.
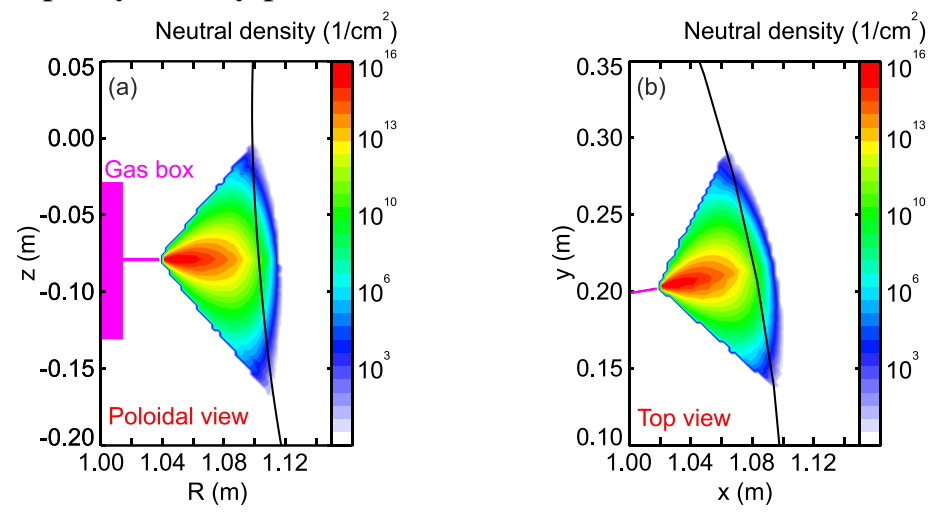

Figure 7. Poloidal and top view of the distribution of first generation of gas puff neutrals obtained with FIDASIM.

\section{Conclusions}

The HFS edge CXRS diagnostic at ASDEX Upgrade has been upgraded with a new fast valve and a new poloidal optical head. The system has been successfully commissioned and now provides impurity temperature, rotation and radiance profiles with high accuracy (up to $5 \mathrm{~mm}$ ). The temperature profile obtained with the upgraded system agrees with measurements from the LFS CXRS systems. A gas puff model has been included in the FIDASIM code to simulate the neutral distribution at the HFS. The high spatial and temporal resolution and the detailed characterization of the flowrate of the upgraded system are the key elements for studying poloidal impurity asymmetries in detail.

\section{Acknowledgments}

The author would like to thank H. Köhnlein, D. Bösser, W. Zeidner, A. Bolland, and M. Rolffs for their valuable help. This work received funding from the VI Plan Propio de Investigación de la Universidad de Sevilla (PPITUS 2017). This work has been carried out within the framework of the EUROfusion Consortium and has received funding from the Euratom research and training programme 2014-2018 and 2019-2020 under grant agreement No 633053. The views and opinions expressed herein do not necessarily reflect those of the 
European Commission. The support from the H2020 Marie-Skłodowska Curie programme (Grant No. 708257) and the Spanish Ministry of Economy and Competitiveness (Grant No. FJCI-201422139) is gratefully acknowledged.

\section{References}

[1] R.J. Fonck et al., Determination of plasma-ion velocity distribution via charge-exchange recombination spectroscopy, Phys. Rev. A 29 (1984) 6.

[2] T. Pütterich et al., Poloidal asymmetry of parallel rotation measured in ASDEX Upgrade, Nucl. Fusion 52 (2012) 083013.

[3] E. Viezzer et al., Rotation and density asymmetries in the presence of large poloidal impurity flows in the edge pedestal, Plasma Phys. Control. Fusion 55 (2013) 124037.

[4] R.M. Churchill et al., In-out impurity density asymmetry in the pedestal region of Alcator C-Mod, Nucl. Fusion 53 (2013) 122002.

[5] R.M. Churchill et al., Development of the gas puff charge exchange recombination spectroscopy (GP-CXRS) technique for ion measurements in the plasma edge, Rev. Sci. Instrum. 84 (2013) 093505.

[6] M. Griener et al., Fast piezoelectric valve offering controlled gas injection in magnetically confined fusion plasmas for diagnostic and fuelling purposes, Rev. Sci. Instrum. 88 (2017) 033509.

[7] https://www.piezosystem.com/products/piezo_actuators/x_nanopositionier/px_500

[8] https://www.nikonmetrology.com/en-us/product/mcax

[9] E. Viezzer et al., Collisionality dependence of edge rotation and in-out impurity asymmetries in ASDEX Upgrade H-mode plasmas, Nucl. Fusion 55 (2015) 123002.

[10] M. von Hellermann et al., Analytical approximation of cross-section effects on charge exchange spectra observed in hot fusion plasmas, Plasma Phys. Control. Fusion 37 (1995) 71.

[11] R. E. Bell and E. J. Synakowski, New understanding of poloidal rotation measurements in a Tokamak plasma, AIP Conf. Proc. 547 (2000) 39.

[12] W. M. Solomon et al., Extraction of poloidal velocity from charge exchange recombination spectroscopy measurements, Rev. Sci. Instrum. 75, 10 (2004) 3481.

[13] W. W. Heidbrink et al., A Code that Simulates Fast-Ion $D_{\alpha}$ and Neutral Particle Measurements, Commun. Comput. Phys. 10 (2011) 716.

[14] B. Geiger, PhD thesis, LMU München (2013). 